\title{
DAFNE as Test Facility for future colliders
}

\author{
Antonio De Santis*广 \\ INFN - Laboratori Nazionali di Frascati \\ E-mail: antonio.desantis@lnf.infn.it
}

DAФNE is a lepton collider working at the energy of the $\phi$-meson resonance, it became operational in 2000. It provided data in consecutive data-taking periods for the KLOE, DEAR and FINUDA experiments until 2006 [1]. Since 2007 it implements a new interaction scheme [2], the so-called "Crab-Waist" collision scheme. It is the only machine that had successfully operated this collision scheme with and without large experiment with solenoids in 2009 with the SIDDHARTA experiment and between 2014 and 2018 with the KLOE-2 experiment, respectively [3].

It will continue operating for PADME (just using the upgraded Linac) and for Siddharta-2 in 2019-2020.

Currently only few accelerator test facilities exists: the ATF2 (KEK), a top-class facility designed for the development of the International Linear Collider, CLASSE(Cornell Laboratory for Accelerator Based Science and Education), a centre of excellence in the development of accelerator technologies located in an university campus, and ANKA (Karlsruhe), devoted to R\&D of machines and applied research. The proposal of DAФNE as a test facility (DAФNE-TF) [5] for accelerator physics and technology development has been presented as an open call for ideas culminated in the organization of a topical workshop in December 2018 [6].

Several lines of technological research have been identified so far for DAФNE-TF: i) Study of low SEY (Secondary Electron Yield) elements and impedances, graphitization of chambers and other surface treatment technologies; ii) New components for accelerators (vacuum chambers, collimators, masks, kickers) and innovative beam diagnostic techniques; iii) Accelerator components realized with 3D printers; iv) High power solid state RF amplifiers; v) Wide-excursion adjustable permanent magnets; vi) High-power positron sources; vii) Components for future SLED and pulse flatness compensation; viii) Emittance manipulators; ix) Beams interacting with amorphous materials, crystals, lasers, plasma; $x$ ) Testing new methods to generate terahertz coherent radiation.

In this contribution, a selection of the different activities proposed during a dedicated workshop held in Frascati Laboratories in December 2018 will be summarized.

European Physical Society Conference on High Energy Physics - EPS-HEP2019 -

10-17 July, 2019

Ghent, Belgium

\section{*Speaker.}

${ }^{\dagger}$ A special thanks to the DAFNE-TF Workshop 2018 advisors and organizers: L. Rivkin (EPFL and PSI), C. Bloise (INFN-LNF), Y. Cai (SLAC), A. Ghigo (INFN-LNF), M. Giovannozzi (CERN), C. Milardi (INFN-LNF), N. Pastrone (INFN-Torino), A. Variola (INFN-LNF) O. R. Blanco Garcia (INFN-LNF), S. Caschera (INFN-LNF), A. De Santis(INFN-LNF), A. Drago (INFN-LNF, chair) 


\section{Introduction}

DAФNE is an electron-positron collider became operational in 2000 [1]. The DAФNE accelerator complex, shown in fig. 1 , includes $50 \mathrm{~Hz}$ repetition rate LINAC including two test beam lines and the corresponding experimental halls, a small dumping ring to improve beam energy spread and emittance before injecting in the two main rings located in the original ADONE dome and lastly a complicated system of transfer lines that distribute the beams to the different complex areas. DAFNE provided data in consecutive data-taking periods for the KLOE, DEAR and FINUDA experiments until 2006. In 2007 a major upgrade program took place with the aim of testing an innovative collision scheme, the "Crab-Waist" [2]. This approach was tested with a physics run for the SIDDHARTA experiment in 2009 and further exploited in 2014-2018 for the KLOE-2 experiment [3, 4], the first experimental setup with strong solenoid using the "Crab-Waist" collision scheme. In both case it provided a considerable increase in terms of instantaneous luminosity.
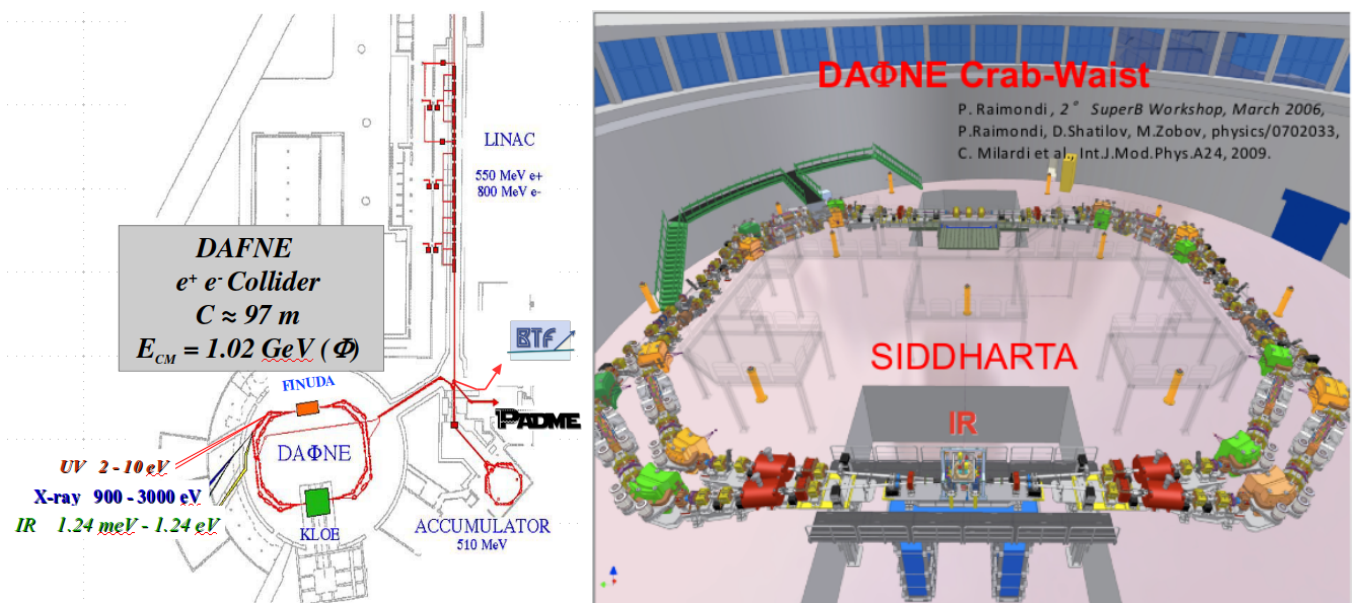

Figure 1: DAFNE accelerator complex (left) and main rings in the Crab-Waist collision scheme configuration (right).

DA $\Phi N E$ will continue operating for PADME (just using the upgraded LINAC) and for SIDDHARTA2 in 2019/2020.

The hardware installed in DA $\Phi N E$, although constantly maintained and improved, dates from mid '90s. A refurbishment focused on the most relevant aspects was realized in 2013, and continued in the following years, while a significant upgrade of the LINAC is in progress, including the doubling of the Beam Test Facility (BTF) in order to increase the number of future users and the installation of a new accelerating section in order to increase the primary beam energy. DAФNE and BTF were able to regularly provide beams for more than 6,000 hours per year, keeping their operational efficiency at levels above $80 \%$ for long periods. During the last decade BTF for external users and DA $\Phi N E$ was simultaneously operated. The complex also powers a synchrotron-light laboratory operated in parasitic mode and a cryogenic plant enabling to operate superconducting magnets and equipments and experimental setups.

In order to exploit the DAFNE potentiality the option of transforming the complex in a test facility for accelerator physics and technology (DAFNE-TF) [5] has been considered and a dedi- 
cated workshop has been held in the Frascati Laboratory in December, 2018. In this contribution a "selected topics" list of received contribution are summarized and reported.

\section{Crystal technologies}

A wide and fruitful area of possible application for DAFNE-TF is the study of interactions between beems and crystals for accelerator technology application. When electron/positron impinges on a crystal with direction nearly parallel to one of the crystal axis or plans its trajectory can be forced in an oscillatory motion within the potential well formed by neighboring axes or planes. This channeling condition leads to a specific e.m. radiation emission, named Channeling Radiation (CR), which is a bit softer but more intense than Coherent Bremsstrahlung (CB). With the $0.5 \mathrm{GeV}$ DAФNE beam an intense $\mathrm{CR}$ around $1 \mathrm{MeV}$ is expected. For comparison, hundreds keV $\mathrm{X}$-rays as synchrotron radiation require a few-GeV electron beam. Even if $\mathrm{CR}$ possesses a strong potential due to its high-intensity, it is not currently exploited for applications due to the spoiling contribution of dechanneling, that limits the radiation intensity and monochromaticity. Since the dechanneling probability is much lower for positive particles and DAФNE is one of the few facilities worldwide providing positrons, this open the way for the realization of an intense CR source at LNF. Moreover, intense e.m. radiation can be generated in periodically bent crystals (Crystalline Undulators-CU), in which the nearly sinusoidal motion of charged particles causes the generation of X-ray ( $\sim 100 \mathrm{keV}$ at DAФNE) with high monochromaticity. The usage of positron beam could lead for the first time to the generation of intense $\mathrm{CU}$ radiation. This kind of source could be high cost effective with respect to present day technology[7].

Concerning the usage of crystals to steer beams the option of extracting the positron beam could be realized using bent crystal in order to obtain a high-quality spill of with good energy spread and emittance smaller. The high value of the duty-cycle, expected in a non-resonant, crystal based extraction scheme, should also guarantee a very flat extracted flux and strong reduction of the background and of the pile-up in the experiments using it. A bent-crystals properly oriented intercepts the diffusive halo, steadily sustained with transverse random noise injection, and kicks it with the crystal inter-planar potential. This non-resonant technique will provide a continuous multi-turn extraction with high-efficiency, better spill quality and simpler and cheaper way than standard non-resonant extraction. At the same time, crystals can support the resonant approach strongly contributing to extract particles towards the extraction septum, realizing a crystal-resonant hybrid extraction [8].

\section{Vacuum equipment and surface treatment}

DAFNE-TF offers a unique opportunity to test vacuum components and surface modifications with beams. Thanks to its flexible beam parameters, a large spectrum of applications can be covered and a multitude of experiments could be conceived. A typical example is the study of photon interaction with surfaces exposed to synchrotron radiation. Indeed, DAFNE may provide photons, in either a large or narrow spectrum of energy, that can be focused on samples or distributed along metre-long vacuum vessels. In the framework of HL-LHC and FCC, CERN's Technology Department has a running collaboration with INFN on this theme. The effect of specific surface 
treatments on electron multipacting can be measured with positively charged beams. In that respect, DAFNE-TF would be the ideal test bench to assess surface modifications and validate new concepts for electron pickups and other sensors. New designs and materials could be tested for impedance reduction at different temperatures with beams having distinct characteristics. In addition to the exceptional beam quality of DAFNE, LNF is equipped with key infrastructures, e.g. cryogenic facilities, and provides the expertise of skilled technical and scientific colleagues; both are essential for the success of those experiments [9].

DAFNE-TF could also contribute to a deeper understanding of the beam heat load on vacuum equipments, essential for the successful performance of circular colliders using superconducting magnets, in fact a beam heat load to superconducting magnets higher than predicted implies a lower magnetic field seen by the beam or even a complete failure of the machine in case of magnet quenching. In a first stage COLDDIAG, a cold vacuum chamber for diagnostics equipped with heaters and temperature sensors, retarding field analyzers, pressure gauges, residual gauge analyzers as well as by a solenoid with an on axis field of $10 \mathrm{mT}$ in the middle of the magnet, could be installed. The test set up could be further expanded using the BEam Screen Testbench EXperiment (BESTEX) setup with cryogenic vacuum chamber insertion flexible enough to exchange cold bores with different geometries and surfaces and be adaptable in different accelerator. In this context an upgraded BESTEX test chamber that could also be tested with electron and, what is more important, with positron beams at DAFNE-TF [10].

\section{Feedback techniques $R \& D$}

New high-gain transverse instability feedback control methods for large rings or extremely high growth rates are strongly needed because the existing control schemes commonly used have limitations from noise in the pickup and processing as well as group delay limits from the processing techniques which look problematic for future machines such as FCC. The development of new processing methods is possible by using multiple pickups (at unique betatron phases) to lower the noise and calculate high-gain correction signals in a single turn of latency. The goals would be both the development of low-noise processing as well as new algorithms for computation. These methods could be tried on DAFNE-TF and quantifying the performance limits of existing and new methods in a careful way, resulting in publications and demonstration technology that can guide new technology efforts. The scale of this can be modest (mostly built on the existing processing at DAFNE with judicious use of available pickups, revised firmware) or if resources allow technology development a complete new architecture and technology platform could be developed and evaluated at DAFNE-TF, with potential application to demonstrations at other facilities [11].

\section{Beam-Beam and collective effects}

Future hadron colliders under study at the Future Circular Collider collaboration plan to collide for the first time protons at energies of 13 and $50 \mathrm{TeV}$ for the High Energy Large Hadron Collider (HE-LHC) and the Future Circular Collider hadron-hadron (FCC-hh), respectively. For the first time the effect of radiation damping become important for the proton dynamics and therefore it has to be included in the beam dynamics models [12]. Present models of the electromagnetic 
interactions between the two colliding beams (so called beam-beam effects) for the Large Hadron Collider do not cover these effects since they are negligible. Due to the higher energies foreseen radiation damping becomes important and has to be modeled and the relevant experience and knowledge should be acquired. Beam-beam models from the LHC will have to be up-dated to take into account such effects (i.e. synchrotron radiation, quantum excitations). The benchmark of the numerical models with the DAFNE collider data represents an important and unique opportunity. The beam-beam parameter for such future colliders is now limited to maximum 0.03 . The study of any possible extension or limitation of such value would be a fundamental input to the design. A list of studies to be performed is: i) Study the beam-beam effects for different radiation damping and for different beam-beam parameters. With and without beam-beam long-range encounters to understand differences and identify scaling laws where possible; ii) Study the coupling of beambeam coherent modes to the machine impedance modes. This coupling has been observed in one single dedicated experiment at the LHC for the case of one single beam-beam interaction. The extension to beam-beam long-range effects is still to be addressed; iii) Effects of a finite crossing angle on the particle dynamics would also provide an important data sample for code benchmarking together with the exploration of the parameter space; iv) DAFNE collider could represent a unique opportunity to collect data to train a model using machine-learning techniques to describe the collider performances in terms of losses and luminosity production. The model could be tested for predicting the performances and would represent an important data sample to possibly compare to LHC models under study at EPFL and CERN.

\section{Muon collider R\&D: LEMMA case}

In the framework of the Low EMittance Muon Collider (LEMMA) R\&D program a very important experimental test could be performed at DAFNE-TF for the positron-ring-plus-target scheme foreseen for LEMMA [13]. This test would be a validation and an experimental benchmarking of the beam dynamics particle tracking studies already on-going. The study implies the usage of different targets, i.e. material and thickness and would require the development of dedicated diagnostic needed to observe the behavior of the circulating beam. Turn-by-turn measurement systems will be needed to measure charge, lifetime and transverse size evolution after multiple interaction with targets. Measurements on the temperature and thermo-mechanical stress on the target will complete the LEMMA test at DAFNE-TF.

\section{Conclusion}

The possible use of DAФNE-TF at the end of its operation as a collider is believed of great interest and useful for the international community of accelerator physicists. Maintaining the experimentation on electron machines would be an asset for LNF and INFN. The functioning, including a partial one, from 2020 onwards, would allow to preserve the expertise of the community of LNF and INFN physicists, technologists, and technicians in electron-based machines, which would represent a resource for the future EuPRAXIA project as well. Without conducting a systematic investigation, a range of technological problems that could be studied at DAФNE-TF, and which are of great interest to the community, have been identified. Moreover the availability of 
this machine would certainly lead to other ideas, whose feasibility should be assessed. There is also space for small fundamental and applied physics experiments, besides the measurements with synchrotron light, which further justifies the operation of the machine for a few months a year, without overburdening on costs and personnel engagement. In order to take full advantage of such opportunities, a plan of consolidation of some of the most obsolete hardware components, together with the installation of a more advanced diagnostics must be scheduled. In this framework, cooperation agreements with other European laboratories can include the use of equipment provided by the users. A dedicated mini-workshop has been organized to discuss accelerator and users community proposals. The contribution received for the workshop exceeded the time allocated for it proving the large interest that such option triggered in the community. All contribution should be investigated carefully and deeply in order to understand the possible outcome in the framework of a rigorous cost/benefits analysis. A second workshop with more advanced proposal and focused discussion is foreseen in the near future in order to allow the INF and the LNF management to undertake the needed decision.

\section{Acknowledgments*}

As part of the local organizing committee I would like to acknowledge the effort made by all contributors to the DAFNE-TF Workshop: S. Guiducci, M. Boscolo, F. Zimmermann, F. Sirghi, C. Curceanu, J. Zmeskal, J. Keintzel, A. Scordo, O. Malyshev, J. Fox, R. Valizadeh, M. Cestelli Guidi, T. Pieloni, E. Pace, R. Claudi, L. Bandiera, V. Guidi, A. Mazzolari, A. Sytov, S. Dabagov, A. Ghigo, D. Hampai, F. M. Addesa, G. Cavoto, F. Iacoangeli, P. Valente, K. Oide, P. Chiggiato, M. Garattini, A. Mochihashi, P. W. Cattaneo, S. Casalbuoni, A. Davide, J. Byrd.

\section{References}

[1] Gallo A et al. 2006 Conf. Proc. C 060626 604-606 SLAC -PUB-12093.

[2] Zobov M et al. 2010 Phys. Rev. Lett. 104 174801;

[3] Milardi C et al. 2012 JINST 7 T03002.

[4] C. Milardi et al. TUYGBD2, IPAC2018.

[5] Bloise C et al. 2018 Frascati Notes INFN-18-10-LNF.

[6] O. R. Blanco Garcia, A. De Santis (editor), A. Drago(chair), 2018, Conference Book of abstracts. https://agenda.infn.it/event/16334/attachments/23692/26963/booklet.pdf

[7] Bandiera, et al.. DAFNE-TF Workshop contribution

[8] M. Garattin, et al.. DAFNE-TF Workshop contribution

[9] P. Chiggiato, et al.. DAFNE-TF Workshop contribution

[10] S.Casalbuoni, et al.. DAFNE-TF Workshop contribution

[11] J. Fox, et al.. DAFNE-TF Workshop contribution

[12] T. Pieloni, et al.. DAFNE-TF Workshop contribution

[13] M. Boscolo, et al.. DAFNE-TF Workshop contribution (doi:10.1088/1742-6596/1067/2/022013) 